\title{
Improvement of Bone Physiology and Life Quality Due to Association of Risedronate and Anastrozole
}

\section{OPEN ACCESS}

Edited by:

Giovanni Li Volti,

University of Catania, Italy

Reviewed by:

Giuseppe Di Giovanni, University of Malta, Malta

Michele D'Amico,

Università degli Studi della Campania

"L. Vanvitelli" Naples, Italy

*Correspondence:

Giovanni Messina

giovanni.messina@unifg.it

Antonietta Messina

antonietta.messina@unicampania.it

${ }^{\dagger}$ These authors have contributed equally to this work.

Specialty section:

This article was submitted to Experimental Pharmacology and Drug

Discovery,

a section of the journal

Frontiers in Pharmacology

Received: 11 July 2017

Accepted: 28 August 2017

Published: 12 September 2017

Citation:

Monda V, Lupoli GA, Messina G,

Peluso R, Panico A, Villano I,

Salerno M, Sessa F, Marciello F, Moscatelli F, Valenzano A, Molino L,

Lupoli $R$, Fonderico F, Tortora A,

Pisano A, Ruberto M, Gabriella M, Cavaliere G, Trinchese G, Mollica MP,

Cipolloni L, Cibelli G, Monda M,

Lupoli G and Messina A (2017) Improvement of Bone Physiology and

Life Quality Due to Association of

Risedronate and Anastrozole.

Front. Pharmacol. 8:632.

doi: 10.3389/fphar.2017.00632

\begin{abstract}
Vincenzo Monda ${ }^{1 \dagger}$, Gelsy A. Lupoli ${ }^{2 \dagger}$, Giovanni Messina ${ }^{3 *}$, Rosario Peluso ${ }^{1,4}$, Annalisa Panico ${ }^{1}$, Ines Villano ${ }^{2}$, Monica Salerno ${ }^{3}$, Francesco Sessa ${ }^{3}$, Francesca Marciello ${ }^{1}$, Fiorenzo Moscatelli ${ }^{3}$, Anna Valenzano ${ }^{3}$, Leonardo Molino ${ }^{4}$ Roberta Lupoli ${ }^{1}$, Francesco Fonderico ${ }^{1}$, Anna Tortora ${ }^{1}$, Agata Pisano ${ }^{1,5}$, Maria Ruberto ${ }^{6}$, Marsala Gabriella ${ }^{7}$, Gina Cavaliere ${ }^{8}$, Giovanna Trinchese ${ }^{8}$, Maria P. Mollica ${ }^{8}$, Luigi Cipolloni ${ }^{9}$, Giuseppe Cibelli ${ }^{3}$, Marcellino Monda ${ }^{2}$, Giovanni Lupoli ${ }^{1}$ and Antonietta Messina ${ }^{2 *}$

${ }^{1}$ Section of Human Physiology and Unit of Dietetic and Sport Medicine, Department of Experimental Medicine, University of Campania "L. Vanvitelli," Naples, Italy, ${ }^{2}$ Department of Clinical Medicine and Surgery, University of Naples Federico II, Naples, Italy, ${ }^{3}$ Department of Clinical and Experimental Medicine, University of Foggia, Foggia, Italy, ${ }^{4}$ Rheumatology Research Unit, University of Naples Federico II, Naples, Italy, ${ }^{5}$ Department of Oncohematology, Santa Maria delle Grazie Hospital, Pozzuoli, Italy, ${ }^{6}$ Department of Medical-Surgical and Dental Specialties, University of Campania "L.Vanvitelli," Naples, Italy, ${ }^{7}$ Struttura Complessa di Farmacia, Azienda Ospedaliero-Universitaria, Ospedali Riuniti di Foggia, Foggia, Italy, ${ }^{8}$ Department of Biology, University of Naples Federico II, Naples, Italy, ${ }^{9}$ Università degli Studi di Roma La Sapienza, Rome, Italy
\end{abstract}

The endocrine therapy is the new frontiers of many breast cancers hormone sensitive. Hormone therapy for treating women with hormone receptor-positive cancer suppresses breast cancer growth either by reducing estrogen synthesis or by interfering with the action of estrogen within tumor cells. In this prospective randomized observational study we investigate the effect of adjuvant anastrozole in monotherapy or associated with risedronate on bone physiology and quality of life in postmenopausal, hormone-sensitive early breast cancer women at mild to moderate risk of fragility fractures.

Methods : 84 women were randomly assigned to receive anastrozole alone (group A) or anastrozole plus oral risedronate (group A+R). At baseline and after 24 months lumbar spine (LS) and femoral neck (FN) BMD were evaluated with dual-energy $x$-ray absorptiometry and health-related quality of life (HRQoL) was examined using the short-form healthy survey.

Results : After 24 months, the group $A+R$ has showed a significant increase in T-score for LS $(p<0.05)$ and for FN $(p<0.05)$ whereas women of group A had a statistically significant rate of bone loss both in LS T-score $(p<0.05)$ and in FN $(p<0.05)$. A significant change in T-score BMD was seen for group $A+R$ compared with group $A$ at the LS $(p=0.04)$ and at FN ( $p=0.04)$. Finally, group $A+R$ showed an overall significant improvement of health profile (SF-36) in group $A(p=0.03)$.

Conclusion : Postmenopausal breast cancer women with osteopenia during treatment with anastrozole have considerable risk of developing osteoporosis during the first 2 years; preventive measures such as healthy lifestyle and daily supplements of calcium and vitamin $\mathrm{D}$ alone seem to be insufficient in holding their bones healthy. Our findings 
suggest the usefulness of addition of risedronate in order to prevent aromatase inhibitorsrelated bone loss, not only in case of high-risk of fractures, but also for women at mild-moderate risk. This determines a significant improvement in bone health and a positive impact on HRQoL.

Keywords: health-related quality of life, bone density, anastrozole, risedronate, breast cancer

\section{INTRODUCTION}

The endocrine therapy is the new frontiers of many breast cancers hormone sensitive, linked to specific genetic alteration such as copy number aberrations (i.e., ERBB2, CCND1 amplification loci), homozygous deletions of $C D K N 2 A / B$ and $P T E N$, and high-frequency substitution and insertion/deletion (indel) driver mutations in cancer genes like TP53 ( $\sim$ frequency $53 \%$ ), PIK3CA (8-26\%), CDH1 (21\%), AKT1 (8\%) and GATA3 (4\%). Moreover, germline exploration has led to documentation of rare, high-penetrance (BRCA1, BRCA2, TP53), moderate penetrance (PTEN, STK11, CDH1, ATM, CHEK2, BRIP1, PALB2) and common, low penetrance risk alleles for developing breast cancer (Li Volti et al., 2011; Salomone et al., 2013; Tibullo et al., 2013; Pomara et al., 2016; Barone et al., 2017; Hahnen et al., 2017; Nik-Zainal and Morganella, 2017; Rizvi et al., 2017). Hormone therapy for treating women with hormone receptor-positive cancer suppresses breast cancer growth either by reducing estrogen synthesis or by interfering with the action of estrogen within tumor cells (Gradishar and Jordan, 1999). In postmenopausal women, the treatment of breast and ovarian cancer are carried out with aromatase inhibitors (AIs); they effect on the peripheral conversion of androgens to estrogens, acting on cytochrome P450 CYP-19 enzyme (Smith and Dowsett, 2003). The adjuvant treatment of postmenopausal women with hormone receptor-positive early breast cancer was carried out with third generation of AIs, such as anastrozole, exemestane and letrozole that have shown superior efficacy and generally better safety and tolerability profile compared with tamoxifen (Arimidex, 2008). However, the bone metabolism is influenced indirectly and positively by estrogens. Stimulating the production of several cytokines, they act either as inhibitors of osteoclastogenesis or as antireceptive agents leading active osteoclasts to apoptosis: several clinical studies have demonstrated the effects of AIs on bone physiology showing an increase in biochemical markers of bone turnover, a decrease in bone mineral density (BMD) and a consequent increase of risk of fractures (Eastell and Hannon, 2005; Santen, 2011; Di Bernardo et al., 2014; Triggiani et al., 2015). Moreover, skeletal fragility and fracture risk are increased by breast cancer, even in the absence of bone metastases. The secretion of the parathyroid hormone-related protein, the chemotherapy, or premature ovarian failures can mediate these effects (Perez and Weilbaecher, 2006; Marra et al., 2013; Monda et al., 2014; De Fusco et al., 2017; Panico et al., 2017); estrogen deprivation caused by AIs administration can exacerbate this risk. To avoid this adverse event, antiresorptive agents such as bisphosphonates (BPs) can be used in breast cancer and in combination with AIs (Greenspan et al., 2007; Hines et al., 2009;
Van Poznak et al., 2010; Messina et al., 2017). Bisphosphonates are pyrophosphate analogs which contain a phosphate-carbonphosphate (P-C-P) core structure. They have an effect on bone escaping from enzymatic degradation. Risedronate is a pyridinyl bisphosphonate validated for the prevention and treatment of postmenopausal osteoporosis (Valenzano et al., 2016). His efficacy was described in decreasing the rate of bone turnover and reversing the loss of $\mathrm{BMD}$, with an adverse effect profile that is similar to placebo.

In this study we investigate the effect of adjuvant anastrozole in monotherapy or associated with risedronate on bone physiology and quality of life in postmenopausal, hormonesensitive early breast cancer women having pre-existing mild to moderate risk of fragility fractures.

\section{MATERIALS AND METHODS}

This is a prospective randomized observational study in which women were treated for 24 months (Figure 1). Participants were provided with both written and oral information regarding the study protocol and were ensured that they were free to withdraw from the study at any time. Before participation, informed consent were subscribed. All procedures conformed

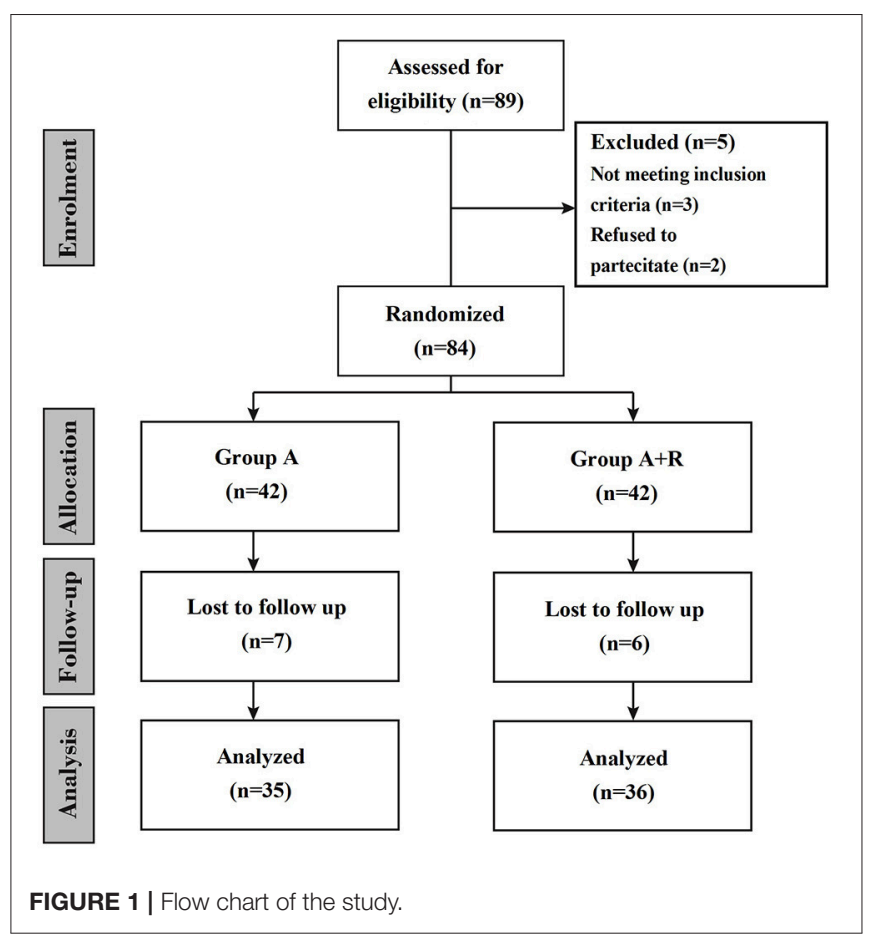


to the directives of the Declaration of Helsinki. This study has been approved by the Azienda Universitaria Policlinico (AUP) of the University of Naples. We enrolled a total of 84 eligible postmenopausal women with hormone receptorpositive breast cancer; they had completed primary surgery and chemotherapy (if indicated) and were categorized according to their baseline BMD T-score as affected by osteopenia (T-score less than-1.0 but greater than or equal to- 2.5 at either the lumbar spine or femoral site) and at mild to moderate risk of fracture. Other clinical criteria associated with T-score were considered: advanced age, early menopause (age $<45$ years), low body weight, current smoking habit, history of fragility fracture in a first-degree relative. Exclusion criteria included menopause induced by prior chemotherapy or any other drug therapy, metastatic disease, recent hormonal treatment, previous hip (HP) fractures or protheses, known bone metabolism disorder, non-treated hypocalcemia, hypercalcemia, previous treatment with selective estrogen receptor modulators (SERMs), hormone-replacement therapy (HRT) or BPs, liver or renal dysfunction. Height, weight, age and medical history were recorded. The primary end-point was to investigate changes in lumbar spine (LS) and femoral neck (FN) BMD at baseline (T0) and after 24 months (T24). Another secondary end-point was to examine risedronate effect on health-related quality of life (HRQoL) using the short-form healthy survey (SF-36). Women were randomly assigned to receive anastrozole plus oral risedronate or anastrozole alone. All enrolled women received supplements of calcium (1,000 mg/day) and vitamin D (800 IU/day). Anastrozole (Arimidex ${ }^{\mathrm{TM}}$; AstraZeneca, London, UK) was given at a dosage of $1 \mathrm{mg}$ /day while oral risedronate (Actonel; Sanofi-Aventis, Paris, France) was given at $35 \mathrm{mg} /$ week. Because oral bisphosphonates are poorly absorbed and for the potential gastrointestinal toxicity, such as esophagitis and esophageal ulcers or erosions, we recommended women to take risedronate early in the morning before taking any food, drink or medicines, remaining upright for at least $30 \mathrm{~min}$ (Crandall, 2001; Markopoulos et al., 2010). In our study risedronate therapy was carefully planned and appropriate preventive measures were taken to minimize the risk of developing osteonecrosis of the jaw (Papapetrou, 2009; Silverman and Landesberg, 2009). These include a complete dental examination with preventive dentistry before initiating risedronate, avoiding invasive dental procedures whenever possible, and recommending our women to maintain an excellent oral hygiene (Diel et al., 2007). The BMD was measured at both the LS and FN at baseline and 24 months later (T24) by dual energy x-ray adsorptiometry (DXA) by the same operator and the same densitomer (DXA QDR 1000; Hologic, Waltham, MA, USA). T-scores were determined according to the World Health Organization (WHO) definition as standard deviation (SD) units from the mean BMD of 25-year-old healthy women (Cohen, 2010). Furthermore, women underwent lateral radiographs and digital computerized morphometry (DCM) of thoracic and lumbar spine at T0 and T24. Vertebral fractures were defined as a decrease of more than or equal to $20 \%$ in any of vertebral heights (anterior, central, and posterior) (Pedrazzoni et al., 2003). Finally, we administered to the women at each visit the SF-36. This validated questionnaire was used to measure the HRQoL, being the most popular generic health status measure due to its comprehensiveness, shortness and high levels of reliability and validity. The SF- 36 contains 36 questions, which take on average $10 \mathrm{~min}$ to be answered; it includes eight health concepts: physical functioning $(\mathrm{PF})$, role limitations due to physical problems (RP), bodily pain (BP), general health perceptions $(\mathrm{GH})$, vitality $(\mathrm{VT})$, social functioning $(\mathrm{SF})$, role limitations due to emotional problems (RE) and mental health $(\mathrm{MH})$. Each concept is assessed using a multi-item scale; item scores are summed for each scale and transformed on a scale of 0 to 100 , with 0 and 100 corresponding to the poorest and optimal health statuses, respectively. The eight scores design a health profile which is a useful and intuitive tool to describe the HRQoL of a group of individuals or a population. In a second step it is possible to calculate two summary measures which reasume the two major domains of the SF-36: the physical component summary (PCS-36) and the mental component summary (MCS36) measure. These indexes have the advantage of being easily used, keeping the good properties of the SF-36 and reducing the number of statistical tests necessary for the eight SF-36 scales; they allow a better interpretation of the results (Jenkinson et al., 1993; Liberman et al., 1995; Ware et al., 1995). In this study, SF-36 was applied by one of the investigators as an interview at baseline and at 84 follow-up visits, also offering help by reading and explaining the questions if necessary. The incidence of adverse events and compliance to treatment were recorded at followup visits during the study. Data are expressed as mean $\pm \mathrm{SD}$ or percentage changes from baseline. Changes from baseline in BMD and SF-36 scores were evaluated in both groups of women using a paired $t$-test while unpaired $t$-test was used to examine the change from baseline to 24 months in BMD between the $\mathrm{A}+\mathrm{R}$ and A groups. A $P$-value of less than 0.05 was considered significant.

\section{RESULTS}

A total of 84 women entered the study; randomly among them 42 women were assigned to receive anastrozole + risedronate (group $\mathrm{A}+\mathrm{R}$ ) and 42 to receive anastrozole alone (group A). A total of 13 women were lost during follow-up, therefore 36 women of group $\mathrm{A}+\mathrm{R}$ and 35 women of group A completed the study (Figure 1). Baseline characteristics and demographics were similar among the two groups, baseline characteristics are summarized in Table 1.

For BMD measurements, mean LS T-score was $-2.04 \pm 0.43$ and mean FN T-score was $-1.80 \pm 0.69$ at baseline in A+R group; in group A mean LS and FN T-score were $-2.06 \pm 0.46$ and $1.98 \pm 0.37$, respectively (Table 1 ). The group $A+R$ has showed a significant increase in T-score from baseline to 24 months with an estimated percentage change for LS of $+6.86 \%(p<0.05)$ and for FN of $+2.8 \%(p<0.05)$. From T0 to T24 women of group A had a statistically significant rate of bone loss estimated at $-4.8 \%(p<$ 0.05 ) in LS T-score and a mean significant decrease in FN T-score of $-3.5 \%(p<0.05)$. Therefore, at 24 month follow-up, T-score $\mathrm{BMD}$ was significantly different for group $\mathrm{A}+\mathrm{R}$ compared with group A both at the lumbar spine $-1.9 \pm 0.49$ vs. $-2.16 \pm 0.51$ 
TABLE 1 | Baseline characteristics of women of group $A+R$ (treated with anastrozole plus oral risedronate) and of group A (anastrozole alone).

\begin{tabular}{lccc}
\hline Characteristics & Group A+R & Group A & p-value* \\
\hline $\mathrm{N}^{\circ}$ & 42 & 42 & \\
mean age $\pm \mathrm{SD}$ & $55.7 \pm 6.4$ & $56.1 \pm 6.3$ & 0.774 \\
height, (cm) mean $\pm \mathrm{SD}$ & $159 \pm 8$ & $162 \pm 9$ & 0.110 \\
weight, (Kg) mean $\pm \mathrm{SD}$ & $58.0 \pm 11.2$ & $60.0 \pm 6.3$ & 0.316 \\
$\mathrm{BMl},\left(\mathrm{Kg} / \mathrm{m}^{2}\right.$ ), mean $\pm \mathrm{SD}$ & $23.4 \pm 2.6$ & $24.0 \pm 2.7$ & 0.303 \\
Mean T-score of lumbar spine & $-2.04 \pm 0.43$ & $-2.06 \pm 0.46$ & 0.838 \\
Mean T-score of femoral neck & $-1.80 \pm 0.69$ & $-1.98 \pm 0.37$ & 0.140
\end{tabular}

*p value by sample T-test.

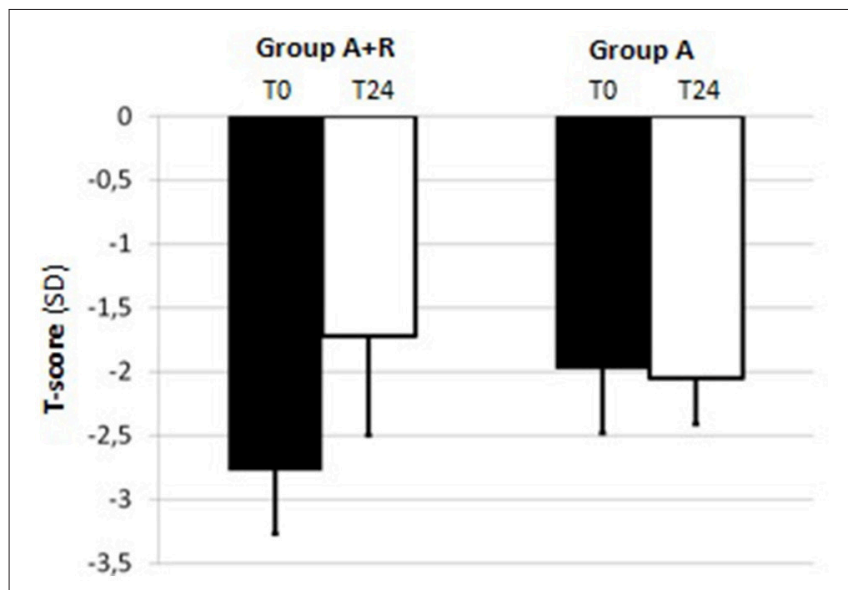

FIGURE 2 | Mean T-score of lumbar spine (LS) at baseline (TO) and at the end of the study (T24) in group $A+R$ (treated with anastrozole plus oral risedronate) and in group A (anastrozole alone).

$(p=0.04)$ and at femoral neck $-1.72 \pm 0.78$ vs. $-2.05 \pm 0.36(p=$ $0.04)$. Among $A+R$ women, only 2 (5.2\%) became osteoporotic, whereas 12 (46\%) in group A moved to the osteoporotic BMD region. Mean percentage changes in LS and FN BMD throughout the study period in both groups are reported in Figures 2, 3.

At baseline, 4 of the enrolled women of group $A+R$ and 2 of group A showed vertebral a traumatic fractures; at T24 none of group $A+R$ occurred in new fragility fractures while among women of group A 3 reported a new fragility fracture (only 1 of these had a previous a traumatic fracture).

HRQoL evaluation evidenced that at baseline the SF-36 scores were $39.8 \pm 9.7$ points in group A and $41.5 \pm 12$ points in group $\mathrm{A}+\mathrm{R}(p>0.05)$. At baselinePCS-36 score was $38.2 \pm 16$ in group $\mathrm{A}+\mathrm{R}$ and in group A was $36.2 \pm 15.3(p>0.05)$ whereas MCS-36 score was $37 \pm 14$ in group $\mathrm{A}$ and $35.9 \pm 15$ in group $\mathrm{A}+\mathrm{R}(p>$ 0.05).

At the end of the study, mean scores in PCS-36 and MCS36 were, respectively, $40.7 \pm 16$ and $38.6 \pm 16$ in group $A+R$ while $38 \pm 15$ and $39.9 \pm 10$ in group A. It was evident that after 24 months the group A+R showed an overall significant improvement of health profile: SF-36 score was $48.6 \pm 7.3$ that is higher than in group A $(44.8 \pm 6.4 ; p=0.03)$ (Figure 4).

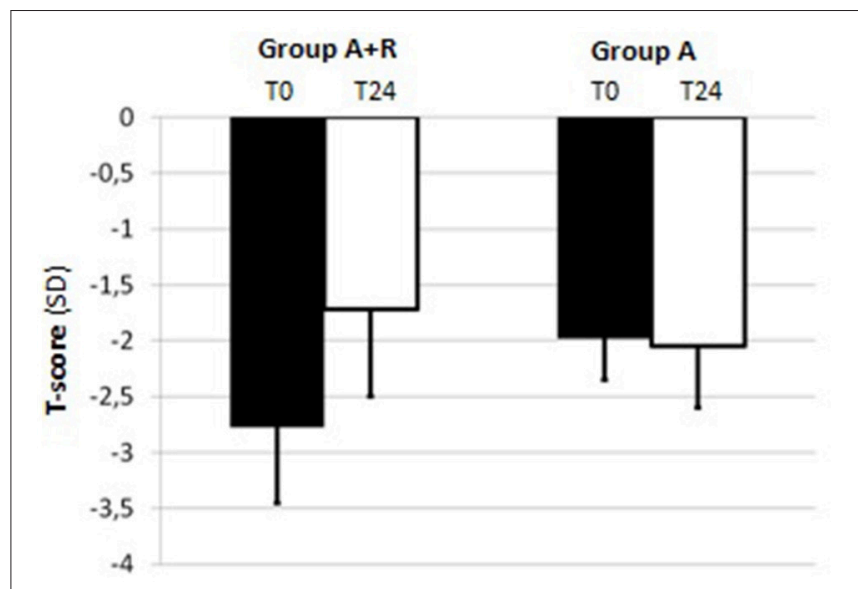

FIGURE 3 | Mean T-score of femoral neck (FN) at baseline (TO) and the end of the study (T24) in group $A+R$ (treated with anastrozole plus oral risedronate) and in group A (anastrozole alone).

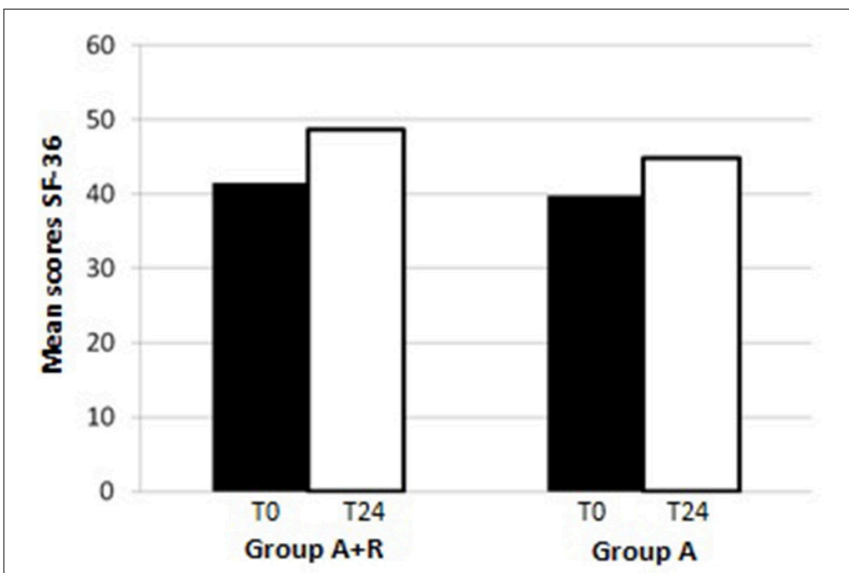

FIGURE 4 | Mean scores of SF-36 at baseline (T0) and after 24 months (T24)in group A+R (treated with anastrozole plus oral risedronate) and in group A (anastrozole alone).

Specifically, a difference was evident in the physical component (Figure 5): there was a higher positive change in women who were treated with $\mathrm{A}+\mathrm{R}(+6.5 \%)$ than in group $\mathrm{A}$ in which this percentage change was $+5.0 \%$. In MCS-36 percentage changes observed were $+7.5 \%$ and $+7.9 \%$ in group $A+R$ and group $A$, respectively (Figure 5).

Treatment was generally well tolerated and adverse events were comparable among groups, having similar frequency. Specifically, 1 patient of group $A+R$ and 2 of group A stopped the treatment for allergic skin reaction and 2 of the $A+R$ for upper gastrointestinal tract symptoms (Figure 1). Other common transient events were mild gastrointestinal tract symptoms such as abdominal pain, indigestion, nausea, diarrhea, and constipation ( 8 in group $A+R$ and 2 in group $A$, respectively). Moreover, 21 women of group A referred musculoskeletal symptoms: 16 women reported arthralgia, 7 myalgia, 4 arthritis, 2 tendinitis and 3 polyalgic syndrome. No case of osteonecrosis 


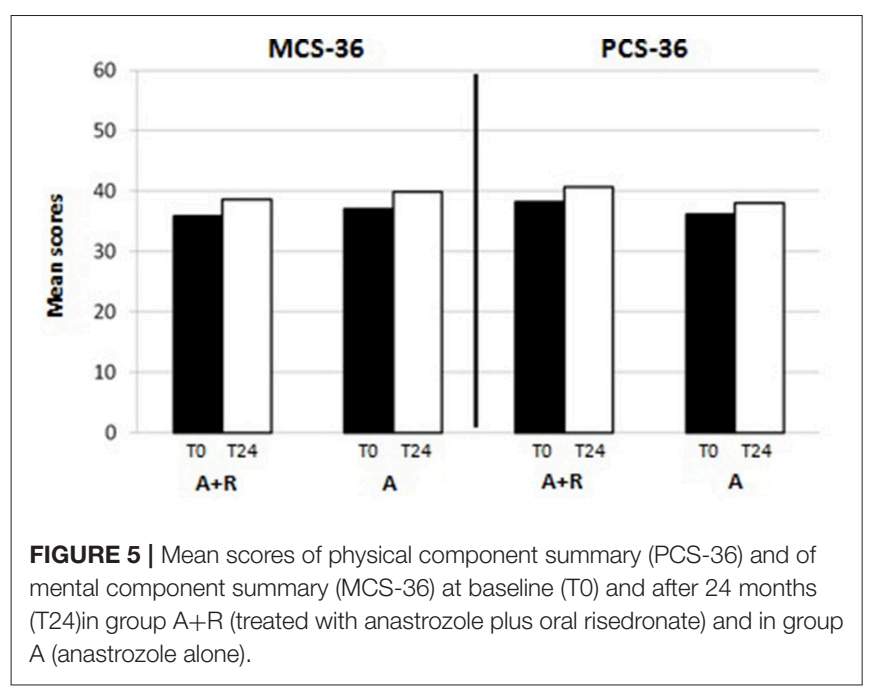

of the jaw was observed in any patient on risedronate treatment during the study period. Compliance to treatment was confirmed during the interview in each patient's visit; occasionally, missed tablets of risedronate were reported by less than $5 \%$ of the women under study treatment.

\section{DISCUSSION}

Our study, aimed at evaluating the effects of risedronate plus adjuvant AIs therapy on bone health and health status in osteopenic postmenopausal early breast cancer women, demonstrates the usefulness of bisphosphonates in inducing a significant improvement of both lumbar-femoral BMD and HRQoL.

The majority of breast cancers are considered hormonesensitive due to the presence of estrogen and/or progesterone receptors; for this reason therapeutic strategies interfering with hormone-mediated tumorgenesis have become a cornerstone of breast cancer management paradigm.

The effect of anastrozole and risedronate in menopausal women affected by breast cancer may be also intended as synergistic suggesting to consider it as an interesting clinical management strategy. Moreover, we could speculate that both drugs may act also inhibiting cytokines pathways such as IL6R/IL6-Notch pathways switches and IL2 and/or increasing levels of proinflammatory cytokines such as IFN-gamma, IL12 , and decreasing levels of IL-4, IL-10 secretion. On the other hand, risedronate may be effective for MMP9-expressing AS by targeting immunosuppressive cells such as M2 macrophage and could increase osteoprotegerin and reduce RANKL and IL-1beta levels.

Comparing aromatase inhibitor therapy with tamoxifen in postmenopausal women with estrogen receptor-positive early breast cancer, it was demonstrated a superior efficacy for AIs treatment; this is quickly becoming the gold standard method. However, the residual estrogens are depleted by AIs treatment and this therapy is frequently associated with rapid bone loss and increased fracture risk.
The first study showing a decrement of BMD in anastrozoletreated women was the prospective bone sub-protocol of the ATAC trial (Marra et al., 2013) in which it was demonstrated a bone loss of $-3.87 \%$ at LS BMD and $-3.92 \%$ total HP BMD and a significantly higher incidence of fracture using anastrozole (7.1\%) than using tamoxifen $(4.4 \%)$ after 2 years' therapy (Ware et al., 1998; Arimidex, 2006). In recent years some studies have used bisphosphonates in order to avoid aromatase inhibitorassociated bone loss in osteoporotic postmenopausal early breast cancer women. Greenspan et al. (2008) examined positive effect of risedronate in women with or without AIs therapy: after 2 years, in risedronate + AIs group, BMD decreased by $-2.4 \%$ at spine and was stable at the hip while women on placebo plus AIs had a mean decrease in BMD of $-4.8 \%$ at the spine and $2.8 \%$ at the total hip; in the placebo without AIs group, BMD was stable at the spine, and had a $1.2 \%$ loss at the total hip and in risedronate without AIs group showed an improvement in BMD of $+2.2 \%$ at the total hip (Howell et al., 2005). The SABRE (Study of Anastrozole with the Bisphosphonate Risedronate) demonstrated that in postmenopausal women with breast cancer who are receiving adjuvant anastrozole and having risk of fragility fracture, the use of risedronate led to a $1-3 \%$ increase in LS BMD and a $1-2 \%$ increase in total HP BMD during a period of 24 months (Van Poznak et al., 2010). Therefore, the beneficial effect of bisphosphonates in reducing bone loss in women affected by osteoporosis has been well described. Instead, the use of bisphosphonates in osteopenic postmenopausal early breast cancer women in AIs therapy is still under debate; in fact, in this case the medical community suggests general preventive measures such as a healthy lifestyle and an adequate intake of calcium and vitamin D. In this context our study could be useful, since it evaluates the use of bisphosphonates as supportive therapy to prevent osteoporosis.

Favorable effects of bisphosphonates on BMD in osteopenic postmenopausal early breast cancer women receiving anastrozole were shown by Markopoulos study (Markopoulos et al., 2010). Women were stratified into those with normal BMD or mild osteopenia $(\mathrm{T}>-2)$ receiving anastozole-only $(\mathrm{A})$ and women with mild or severe osteopenia $(\mathrm{T} \leq-2)$ or osteoporosis $(\mathrm{T}<-2.5)$ receiving anastrozole and per os risedronate $(A+R)$. Depending on age of treatment initiation, women were grouped into two age cohorts, above and below 65 years. Among women receiving A-only, women $\leq 65$ years were more likely to have a decrease in LS-BMD than older ones. HP-BMD decrease at 12 months was not related to age. In women with mild severe osteopenia or osteoporosis, treated with $\mathrm{A}+\mathrm{R}$, no age effect was observed for LS or HP; among women with normal BMD at baseline, the age effect on LS-BMD change was deeper. This study suggested that younger postmenopausal women with normal BMD or mild osteopenia receiving A-only have an increased risk of bone loss in LS. Among women with mild severe osteopenia or osteoporosis treated with $\mathrm{A}+\mathrm{R}, 12$ months $\mathrm{LS}$ or $\mathrm{HP} \mathrm{BMD}$ variations were configured regardless of age group.

Our results demonstrate that the addition of $35 \mathrm{mg}$ oral risedronate weekly to anastrozole improves BMD both at lumbar spine $(+6.8 \%)$ and at femoral neck $(+2.8 \%)$ after 24 months in osteopenic postmenopausal early breast cancer women; instead, in osteopenic women who receive anastrozole alone 
a significant bone loss $(-4.5$ and $-3.5 \%$ at spine and femoral neck, respectively) is shown. We observed, in fact, that $46 \%$ of osteopenic women developed osteoporosis after 2 years of anastrozole treatment. Our findings suggest, therefore, that risedronate may be used in order to prevent further bone loss in women with mild to moderate osteopenia who have to start adjuvant therapy with AIs in breast cancer.

Major trials have established that AIs improve both diseasefree survival and HRQoL and their use as adjuvant endocrine therapy for postmenopausal women with $\mathrm{HR}+$ breast cancer has been steadily increasing. Infact, AIs are also an appealing alternative to tamoxifen because despite their adverse events associated with the menopause-related decline in estrogen level, their use has never been associated with the rare, yet serious, adverse events reported using tamoxifen (e.g., 194 endometrial cancer and venous thromboembolism) (Howell et al., 2005). It should, however, be emphasized that AIs, by reducing plasma estrogens in postmenopausal women, exacerbate bone loss and musculoskeletal symptoms such as arthralgia, myalgia, joint symptoms and polyalgic syndrome. A significant association between BMD values and musculoskeletal symptoms was reported by Muslimani et al. (2009); in this study, it was evidenced that women on AIs developing osteoporosis were at increased risk of musculoskeletal symptoms and bone fracture. However, it was demonstrated that the use of bisphosphonates, in postmenopausal early breast cancer women in therapy with AIs, is associated with adjunctive analgesic benefits, offering these women some relief from arthralgia (Boonen et al., 2004; Pavlakis et al., 2005). In our experience, women treated with anastrazole evidenced severe musculoskeletal symptoms, such as arthralgia, myalgia and arthritis, whereas none of group $A+R$ referred pain of musculoskeletal system. Despite this, little attention has been paid so far to the effect of the use of bisphosphonates on quality of life of these women.

Infact, the novelty of our study is the evaluation of the effect of risedronate addition to adjuvant therapy with AIs on HRQoL. Using SF-36 questionnaire, we observed there was an overall improvement in whole health profile with a slightly higher improvement of physical component in women receiving $A+R$ than in anastrozole group. In fact, the women of this group judged their daily activities (bath, dress, both walk and climb) fairly good; moreover, they reported reduction of pain. These data are quite important since in such women quality of life is an important part of treatment beyond the efficacy indicators.

After all, BPs can play a direct antitumor activity with an added beneficial effect; specifically, it was demonstrated that risedronate and its structural analogs inhibit tumor cell invasion of breast carcinoma cells both in vitro and in vivo. Therefore, in the cases of women cancers that frequently metastasize to

\section{REFERENCES}

Arimidex, T. (2006). Comprehensive side-effect profile of anastrozole and tamoxifen as adjuvant treatment for early-stage breast cancer: longterm safety analysis of the ATAC trial. Lancet Oncol. 7, 633-643. doi: $10.1016 /$ S1470-2045(06)70767-7 bone, BPs may be used as agents for the prophylactic treatment (Boissier et al., 2000; Fournier et al., 2010).

Moreover, tolerability of administration of risedronate and anastrozole in our study was good with few women showing gastrointestinal symptoms and skin reactions. Overall, the safety profiles of anastrozole and risedronate were consistent with those observed in previous clinical studies (White and Perry, 2003; Yamamoto and Iwase, 2008; Briot et al., 2010). No case of ONJ was observed in any patient on risedronate treatment.

In conclusion, women with osteopenic BMD have considerable risk of developing osteoporosis during the first 2 years of treatment with anastrozole; preventive measures such as healthy lifestyle and daily supplements of calcium and vitamin $\mathrm{D}$ alone seem to be insufficient in holding their bones healthy. In our experience, the addition of oral risedronate in postmenopausal breast cancer women in mild to moderate risk region $(-1<\mathrm{T}$-score $<-2.5)$ receiving adjuvant anastrozole gives a significant increase in BMD levels, thus having a very low risk to develop osteoporosis. Moreover, treating women who are on aromatase inhibitors with bisphosphonates also has a significant impact on the patient's quality of life.

Therefore, we suggest the combination of anastrozole plus oral risedronate in order to prevent aromatase inhibitors-related bone loss, not only in postmenopausal breast cancer women at high-risk of fractures, but also for women at mild-moderate risk.

\section{AUTHOR CONTRIBUTIONS}

GAL, VM, RP, AnP, IV, RL, FF, AgP, and MR: conceived the study, participated in its design. MS, FS, FrM, AV, FiM, LM, MG, LC, GCi, and GT: contributed to the conception and design. GAL, VM, GM, and AM: wrote manuscript. AM, MPM, GCa, MM, GL, and GM: drafted the article and revised it critically for important intellectual content; GM and AM: final approval of the version to be published. All authors read and approved the final manuscript.

\section{FUNDING}

This study was supported by grants of Department of Biology, Universitá degli Studi di Napoli Federico II.

\section{ACKNOWLEDGMENTS}

The authors would like to thank Dr Marianna Coppola, Fellow Resercher, Group of Research of Social Interaction, Department of Education, University of Salerno for her support in SF-36 analysis and interpretation; Dr. Robin Lindsay, C.E.L., University of Naples "Orientale" for his contribution in language editing of the text.

Arimidex, T. (2008). Effect of anastrozole and tamoxifen as adjuvant treatment for early-stage breast cancer: 100-month analysis of the ATAC trial. Lancet Oncol. 9, 45-53. doi: 10.1016/S1470-2045(07) 70385-6

Barone, R., Pitruzzella, A., Marino Gammazza, A., Rappa, F., Salerno, M., Barone, F., et al. (2017). Nandrolone decanoate interferes with testosterone biosynthesis 
altering blood-testis barrier components. J. Cell Mol. Med. 21, 1636-1647. doi: $10.1111 /$ jcmm. 13092

Boissier, S., Ferreras, M., Peyruchaud, O., Magnetto, S., Ebetino, F. H., Colombel, M., et al. (2000). Bisphosphonates inhibit breast and prostate carcinoma cell invasion, an early event in the formation of bone metastases. Cancer Res. 60, 2949-2954.

Boonen, S., Haentjens, P., Vandenput, L., and Vanderschueren, D. (2004). Preventing osteoporotic fractures with antiresorptive therapy: implications of microarchitectural changes. J. Intern. Med. 255, 1-12. doi: 10.1046/j.0954-6820.2003.01258.x

Briot, K., Tubiana-Hulin, M., Bastit, L., Kloos, I., and Roux, C. (2010). Effect of a switch of aromatase inhibitors on musculoskeletal symptoms in postmenopausal women with hormone-receptor-positive breast cancer: the ATOLL (articular tolerance of letrozole) study. Breast Cancer Res. Treat. 120, 127-134. doi: 10.1007/s10549-009-0692-7

Cohen, H. V. (2010). Bisphosphonate-associated osteonecrosis of the jaw: patient care considerations: overview for the orthopaedic nursing healthcare professional. Orthop. Nurs. 29, 176-180. doi: 10.1097/NOR.0b013e3181dcd2a2

Crandall, C. (2001). Risedronate: a clinical review. Arch. Intern. Med. 161, 353-360. doi: 10.1001/archinte.161.3.353

De Fusco, C., Messina, A., Monda, V., Viggiano, E., Moscatelli, F., Valenzano, A., et al. (2017). Osteopontin: relation between adipose tissue and bone Homeostasis. Stem Cells Int. 2017:4045238. doi: 10.1155/2017/4045238

Di Bernardo, G., Messina, G., Capasso, S., Del Gaudio, S., Cipollaro, M., Peluso, G., et al. (2014). Sera of overweight people promote in vitro adipocyte differentiation of bone marrow stromal cells. Stem Cell Res. Ther. 5, 4. doi: $10.1186 /$ scrt393

Diel, I. J., Fogelman, I., Al-Nawas, B., Hoffmeister, B., Migliorati, C., Gligorov, J., et al. (2007). Pathophysiology, risk factors and management of bisphosphonateassociated osteonecrosis of the jaw: is there a diverse relationship of aminoand non-aminobisphosphonates? Crit. Rev. Oncol. Hematol. 64, 198-207. doi: 10.1016/j.critrevonc.2007.07.005

Eastell, R., and Hannon, R. (2005). Long-term effects of aromatase inhibitors on bone. J. Steroid Biochem. Mol. Biol. 95, 151-154. doi: 10.1016/j.jsbmb.2005.04.009

Fournier, P. G., Stresing, V., Ebetino, F. H., and Clézardin, P. (2010). How do bisphosphonates inhibit bone metastasis in vivo? Neoplasia 12, 571-578.

Gradishar, W. J., and Jordan, V. C. (1999). Hormonal therapy for breast cancer. an update. Hematol. Oncol. Clin. North Am. 13, 435-455. doi: 10.1016/S0889-8588(05)70064-2

Greenspan, S. L., Bhattacharya, R. K., Sereika, S. M., Brufsky, A., and Vogel, V. G. (2007). Prevention of bone loss in survivors of breast cancer: a randomized, double-blind, placebo-controlled clinical trial. J. Clin. Endocrinol. Metab. 92, 131-136. doi: 10.1210/jc.2006-1272

Greenspan, S. L., Brufsky, A., Lembersky, B. C., Bhattacharya, R., Vujevich, K. T., Perera, S., et al. (2008). Risedronate prevents bone loss in breast cancer survivors: a 2-year, randomized, double-blind, placebo-controlled clinical trial. J. Clin. Oncol. 26, 2644-2652. doi: 10.1200/JCO.2007.15.2967

Hahnen, E., Hauke, J., Engel, C., Neidhardt, G., Rhiem, K., and Schmutzler, R. K. (2017). Germline mutations in triple-negative breast cancer. Breast Care 12, 15-19. doi: 10.1159/000455999

Hines, S. L., Mincey, B. A., Sloan, J. A., Thomas, S. P., Chottiner, E., Loprinzi, C. L., et al. (2009). Phase III randomized, placebo-controlled, double-blind trial of risedronate for the prevention of bone loss in premenopausal women undergoing chemotherapy for primary breast cancer. J. Clin. Oncol. 27, 1047-1053. doi: 10.1200/JCO.2008.19.1783

Howell, A., Cuzick, J., Baum, M., Buzdar, A., Dowsett, M., Forbes, J. F., et al. (2005). Results of the ATAC (Arimidex, Tamoxifen, Alone or in Combination) trial after completion of 5 years' adjuvant treatment for breast cancer. Lancet 365, 60-62. doi: 10.1016/S0140-6736(04)17666-6

Jenkinson, C., Coulter, A., and Wright, L. (1993). Short form 36 (SF36) health survey questionnaire: normative data for adults of working age. BMJ 306, 1437-1440. doi: 10.1136/bmj.306.6890.1437

Li Volti, G., Salomone, S., Sorrenti, V., Mangiameli, A., Urso, V., Siarkos, I., et al. (2011). Effect of silibinin on endothelial dysfunction and ADMA levels in obese diabetic mice. Cardiovasc. Diabetol. 14:62. doi: 10.1186/1475-2840-10-62

Liberman, U. A., Weiss, S. R., Bröll, J., Minne, H. W., Quan, H., Bell, N. H., et al. (1995). Effect of oral alendronate on bone mineral density and the incidence of fractures in postmenopausal osteoporosis. the alendronate phase III osteoporosis treatment study group. $N$ Engl. J. Med. 333, 1437-1444. doi: 10.1056/NEJM199511303332201

Markopoulos, C., Tzoracoleftherakis, E., Polychronis, A., Venizelos, B., Dafni, U., Xepapadakis, G., et al. (2010). Management of anastrozole-induced bone loss in breast cancer women with oral risedronate: results from the ARBI prospective clinical trial. Breast Cancer Res. 12:R24. doi: 10.1186/bcr2565

Marra, L., Cantile, M., Scognamiglio, G., Perdonà, S., La Mantia, E., Cerrone, M., et al. (2013). Deregulation of HOX B13 expression in urinary bladder cancer progression. Curr. Med. Chem. 20, 833-839.

Messina, G., Valenzano, A., Moscatelli, F., Salerno, M., Lonigro, A., Esposito, T., et al. (2017). Role of autonomic nervous system and orexinergic system on adipose tissue. Front. Physiol. 8:137. doi: 10.3389/fphys.2017. 00137

Monda, M., Messina, G., Scognamiglio, I., Lombardi, A., Martin, G. A., Sperlongano, P., et al. (2014). Short-term diet and moderate exercise in young overweight men modulate cardiocyte and hepatocarcinoma survival by oxidative stress. Oxid. Med. Cell Longev. 2014:131024. doi: $10.1155 / 2014 / 131024$

Muslimani, A. A., Spiro, T. P., Chaudhry, A. A., Taylor, H. C., Jaiyesimi, I., and Daw, H. A. (2009). Aromatase inhibitor-related musculoskeletal symptoms: is preventing osteoporosis the key to eliminating these symptoms? Clin. Breast Cancer 9, 34-38. doi: 10.3816/CBC.2009.n.006

Nik-Zainal, S., and Morganella, S. (2017). Mutational signatures in breast cancer: the problem at the DNA level. Clin. Cancer Res. 1, 2617-2629. doi: 10.1158/1078-0432.CCR-16-2810

Panico, A., Messina, G., Lupoli, G. A., Lupoli, R., Cacciapuoti, M., Moscatelli, F., et al. (2017). Quality of life in overweight (obese) and normal-weight women with polycystic ovary syndrome. Patient Prefer Adher 11, 423-429. doi: 10.2147/PPA.S119180

Papapetrou, P. D. (2009). Bisphosphonate-associated adverse events. Hormones 8 , 96-110. doi: 10.14310/horm.2002.1226

Pavlakis, N., Schmidt, R., and Stockler, M. (2005). Bisphosphonates for breast cancer. Cochrane Database Syst. Rev. 20:CD003474. doi: 10.1002/14651858.CD003474.pub2

Pedrazzoni, M., Girasole, G., Bertoldo, F., Bianchi, G., Cepollaro, C., Del Puente, A., et al. (2003). Definition of a population-specific DXA reference standard in Italian women: the Densitometric Italian Normative Study (DINS). Osteoporos. Int. 14, 978-982. doi: 10.1007/s00198-003-1521-1

Perez, E. A., and Weilbaecher, K. (2006). Aromatase inhibitors and bone loss. Oncology 20, 1029-1039.

Pomara, C., Barone, R., Marino Gammazza, A., Sangiorgi, C., Barone, F., Pitruzzella, A., et al. (2016). Effects of nandrolone stimulation on testosterone biosynthesis in leydig cells. J. Cell Physiol. 231, 1385-1391. doi: $10.1002 / j c p .25272$

Rizvi, W., Truong, P., and Truong, Q. (2017). Metastatic breast cancer with BRCA mutation discovered by next-generation sequencing responding to Olaparib. Cureus 11:e1337. doi: 10.7759/cureus.1337

Salomone, F., Barbagallo, I., Puzzo, L., Piazza, C., and Li Volti, G. (2013). Efficacy of adipose tissue-mesenchymal stem cell transplantation in rats with acetaminophen liver injury. Stem Cell Res. 11, 1037-1044. doi: 10.1016/j.scr.2013.07.003

Santen, R. J. (2011). Clinical review: effect of endocrine therapies on bone in breast cancer women. J. Clin. Endocrinol. Metab. 96, 308-319. doi: 10.1210/jc.2010-1679

Silverman, S. L., and Landesberg, R. (2009). Osteonecrosis of the jaw and the role of bisphosphonates: a critical review. Am. J. Med. 122, S33-S45. doi: 10.1016/j.amjmed.2008.12.005

Smith, I. E., and Dowsett, M. (2003). Aromatase inhibitors in breast cancer. N. Engl. J. Med. 348, 2431-2442. doi: 10.1056/NEJMra023246

Tibullo, D., Barbagallo, I., Giallongo, C., La Cava, P., Parrinello, N., Vanella, L., et al. (2013). Nuclear translocation of heme oxygenase- 1 confers resistance to imatinib in chronic myeloid leukemia cells. Curr. Pharm. Des. 19, 2765-2770. doi: 10.2174/1381612811319150012

Triggiani, A. I., Valenzano, A., Ciliberti, M. A., Moscatelli, F., Villani, S., Monda, M., et al. (2015). Heart rate variability is reduced in underweight and overweight healthy adult women. Clin. Physiol. Funct. Imaging 37, 162-167. doi: $10.1111 /$ cpf. 12281 
Valenzano, A., Moscatelli, F., Triggiani, A. I., Capranica, L., De Ioannon, G., Piacentini, M. F., et al. (2016). Heart-rate changes after an ultraendurance swim from Italy to Albania: a case report. Int. J. Sports Physiol. Perform. 11, 407-409. doi: 10.1123/ijspp.2015-0035

Van Poznak, C., Hannon, R. A., Mackey, J. R., Campone, M., Apffelstaedt, J. P., Clack, G., et al. (2010). Prevention of aromatase inhibitor-induced bone loss using risedronate: the SABRE trial. J. Clin. Oncol. 28, 967-975. doi: 10.1200/JCO.2009.24.5902

Ware, J. E. Jr., Gandek, B., Kosinski, M., Aaronson, N. K., Apolone, G., Brazier, J., et al. (1998). The equivalence of SF-36 summary health scores estimated using standard and country-specific algorithms in 10 countries: results from the IQOLA project. international quality of life assessment. J. Clin. Epidemiol. 51, 1167-1170. doi: 10.1016/S0895-4356(98) 00108-5

Ware, J. E. Jr., Kosinski, M., Bayliss, M. S., McHorney, C. A., Rogers, W. H., and Raczek, A. (1995). Comparison of methods for the scoring and statistical analysis of SF-36 health profile and summary measures: summary of results from the medical outcomes study. Med. Care 33, AS264-AS279.

White, N. J., and Perry, C. M. (2003). Risedronate once a week. Treat Endocrinol. 2, 415-420. doi: 10.2165/00024677-200302060-00005
Yamamoto, Y., and Iwase, H. (2008). Safety profiles of aromatase inhibitors and selective estrogen-receptor modulators in the treatment of early breast cancer. Int. J. Clin. Oncol. 13, 384-394. doi: 10.1007/s10147-0080828-5

Conflict of Interest Statement: The authors declare that the research was conducted in the absence of any commercial or financial relationships that could be construed as a potential conflict of interest.

The reviewer MD declared a shared affiliation, with no collaboration, with several of the authors, VM, GM, IV, MR, MM, AM, to the handling Editor.

Copyright (c) 2017 Monda, Lupoli, Messina, Peluso, Panico, Villano, Salerno, Sessa, Marciello, Moscatelli, Valenzano, Molino, Lupoli, Fonderico, Tortora, Pisano, Ruberto, Gabriella, Cavaliere, Trinchese, Mollica, Cipolloni, Cibelli, Monda, Lupoli and Messina. This is an open-access article distributed under the terms of the Creative Commons Attribution License (CC BY). The use, distribution or reproduction in other forums is permitted, provided the original author(s) or licensor are credited and that the original publication in this journal is cited, in accordance with accepted academic practice. No use, distribution or reproduction is permitted which does not comply with these terms. 